\title{
A Dissipative Approach to the Identification of Biochemical Reaction Networks
}

\author{
Dirk Fey* Eric Bullinger* \\ * Systems and Modeling Research Unit, Institute Montefiore, \\ University of Liège, Belgium \\ (email:fey@montefiore.ulg.ac.be, e.bullinger@montefiore.ulg.ac.be)
}

\begin{abstract}
Estimation of kinetic parameters is a key step in modelling biochemical reaction networks as, often, their direct estimation is expensive, time-consuming or even infeasible. This article proposes a parameter estimation procedure, which explicitly takes into account the model structure of the biological systems. The convergence is guaranteed using a dissipativity argument and a coordinate transformation yielding a parameter-free system description. The application to a basic enzyme kinetic model illustrates the proposed methodology.
\end{abstract}

Keywords: identification, state estimation, observers, dissipativity, biochemical systems

\section{INTRODUCTION}

Modelling biological systems on the intracellular level has been a research topic for over half a century. For example, Hodgkin and Huxley [1952] explained the neuron function by means of a mathematical model of different ion channels. Hodgkin and Huxley were able to estimate the model parameters from experimental data, a challenging task still today in most biological systems. Nowadays, ever improving developments of experimental techniques provide more and more high quality experimental data, putting the task of identification into reachable scope. However, the straight forward application of systems theoretical methods to biology is impaired by certain particularities of biological systems. Difficulties concern large numbers of parameters, sparse data and reduced sets of possible stimulations.

Biological systems have peculiar system properties such as for example positivity and monotonicity [Sontag, 2005]. Exploiting these properties has the potential of enhancing current identification techniques. This contribution extends previous research, which proposed the use of observers for estimating kinetic parameters. Here we propose to use state-observers that are based on dissipativity theory for parameter estimation. As in earlier work, known structural information such as stoichiometry and type of reaction kinetics is explicitly used in designing the estimator.

The manuscript is organised as follows. First, Section 2 gives a brief introduction to reaction kinetic modelling of biological systems. Section 3 presents the proposed identification methodology. Section 4 presents a dissipative observer scheme and derives sufficient conditions for efficiently choosing the design parameters of the observer. The applicability of the proposed parameter estimation methodology is illustrates using an enzyme kinetic model (Section 5), before Section 6 summarises the findings.

\section{BIOLOGICAL MODELLING}

A common framework for the modelling of biochemical reaction networks are sets of reactions of the following form

$$
\alpha_{1} \mathrm{~S}_{1}+\ldots+\alpha_{n_{s}} \mathrm{~S}_{n_{s}} \rightarrow \beta_{1} \mathrm{P}_{1}+\ldots+\beta_{n_{p}} \mathrm{P}_{n_{p}},
$$

where $\mathrm{S}_{i}$ denote substrates that are transformed into the products $\mathrm{P}_{i}$. The factors $\alpha_{i}$ and $\beta_{i}$ denote the stoichiometric coefficients of the reactants.

Neglecting spatial and stochastic effects, these reactions are often modelled with systems of ordinary differential equations:

$$
\dot{c}=N v(c, p)
$$

where $c \in \mathbb{R}_{\geq 0}^{n_{c}}$ is the vector of concentrations, $p \in \mathbb{R}_{>0}^{m}$ the parameter vector and $v \in \mathbb{R}_{\geq 0}^{n_{c}} \times \mathbb{R}_{>0}^{m} \mapsto \mathbb{R}_{\geq 0}^{n_{v}}$ the vector of the flows. The stoichiometric matrix $N \in \mathbb{R}^{n_{c} \times n_{v}}$ depends on the coefficients $\alpha_{i}, \beta_{i}$ and, possibly on factors compensating different units or volumina. For a more detailed introduction, see for example Klipp et al. [2005] or Keener and Sneyd [2001].

A variety of kinetics models exist for the reaction rates $v(c, p)$ [Cornish-Bowden, 2004]. Many of them can be written in the following form

$$
v_{i}(c, p)=k_{i} \prod_{j} \frac{c_{j}^{\nu_{i j}}}{M_{i j}}, \quad \text { where } \quad M_{i j}=c_{j}^{\eta_{i j}}+K_{i j}^{\eta_{i j}} .
$$

If $\eta_{i j}=0$, then the arbitrary parameter $K_{i j}$ shall be equal to 1 . The general formulation of $(2 \mathrm{~b})$ contains mass action kinetics, generalised mass action kinetics, MichaelisMenten- and Hill-kinetics as well as their products. For example, setting $\eta_{i j}=0$ leads to mass action kinetics.

We assume that we measure some species concentration and/or reaction rates, or linear combinations thereof, e.g. the sum of all molecules in a particular phosphorylation state. Thus, the output is a (nonlinear) function $\mathbb{R}^{n_{c}} \mapsto$ $\mathbb{R}^{n_{y}}$ 


$$
y=C\left[\begin{array}{c}
c \\
v(c, p)
\end{array}\right],
$$

where $C \in \mathbb{R}^{n_{y} \times\left(n_{c}+n_{v}\right)}$.

In most biological systems, the model structure, i.e. the stoichiometry and the reaction rate models, are known, while the parameters need to be estimated from inputoutput data. This paper proposes a parameter estimation method that utilises the model structure information.

\section{PARAMETER ESTIMATION VIA PARAMETER-FREE COORDINATE SYSTEM}

The proposed parameter estimation method utilises the peculiarities of the model structure. The latter, i.e. $N, \nu$ and $\eta$, are often known while the kinetic parameters $k$ and $K$ are frequently difficult to measure and vary greatly from organism to organism.

In the context of the above stated model class, the problem can be formulated as follows:

Given: The structure, i.e. $N, \nu$ and $\eta$, and continuous time course measurements $y(t)$.

Unknown: The kinetic parameters, i.e. $k$ and $K$.

These unknown parameters need to be estimated from measurements $y(t)$. Ideally, the methodology exploits the known systems structure and leads reliably to accurate estimates.

Farina et al. [2006] proposed the transformation into the parameter independent form, which facilitates the actual estimation process for mass action systems. Fey et al. [2008, 2009] extended the transformation for systems towards Hill-like kinetics of the form (2b) and proposed to use nonlinear state-observers for the estimation. In these previous publications, we developed a three stage parameter estimation, consisting of the following steps:

(1) Transformation of the system of ordinary differential equations (2) into a parameter independent form;

(2) Estimation of all states in the parameter free coordinates;

(3) Calculation of the parameters based on the state estimate.

Previously, the second step consisted of an observer designed in observability normal form. The novelty of this paper is to eliminate the need for this second coordinate transformation by directly designing an observer for the parameter independent system description.

\subsection{Transformation into parameter independent form}

Observer-based approaches to parameter estimation rely on a certain state space extension. Under the assumption of constant parameters, the parameter estimation problem is transformed into a state estimation problem. Considering the extended system the parameters can be estimated using a suitable observer. This section recapitulates the coordinate transformation proposed by Farina et al. [2006] and Fey et al. [2008]. Consider the system (2a) extended by (trivial) differential equations for the parameters:

$$
\begin{aligned}
\dot{c} & =N v(c, p), \\
\dot{p} & =0 .
\end{aligned}
$$

Note 1. The assumption $\dot{p}=0$ is a formal construct that enables to formulate the problem mathematically. The same approach can be taken in order to estimate time varying parameters. Then the assumption $\dot{p}=0$ corresponds to a time scale separation of system dynamics and varying parameters.

Further, assume that the following holds.

Assumption 1. The parameters $p$ and the concentrations $c$ are strictly positive along trajectories of (3) and bounded, i.e. $0<\underline{\delta} \leq c_{i}\left(t, c_{0}\right) \leq \bar{\delta}<\infty$ holds for all species $i$ and all initial conditions $c_{0}$ for some positive constants $\underline{\delta}<\bar{\delta}$.

This condition is satisfied in many biological application, in particular for models of metabolic pathways.

As system (3) depends on unknown parameters, designing an observer is not straightforward and might require an iteration between state estimation and observer adaptation. To avoid that drawback, an equivalent system description can be found using Assumption 1. This descriptions is free of parameters and explicitly uses the known systems structure. The approach is first illustrated for a system with a single reaction, before the general extension scheme is presented.

Example 2. Let us consider the following system

$$
\begin{aligned}
\dot{c} & =-v(c), \\
v(c) & =k \frac{c^{2}}{c+K} .
\end{aligned}
$$

Due to Assumption 1, $c$ and therefore $v$ are positive. It is therefore possible to derive the differential equation for the relative rate of change of the reaction rate, in essence taking the logarithm and time derivative of (4b). Before however we introduce the new state

$$
M=c+K
$$

with the differential equation

$$
\dot{M}=\dot{c} .
$$

Now taking the logarithm and time derivative of $(4 \mathrm{~b})$ gives

$$
\begin{aligned}
\frac{\dot{v}}{v} & =\frac{d}{d t} \log v=\frac{d}{d t} \log \left(k \frac{c^{2}}{M}\right) \\
& =\frac{d}{d t}(\log k+2 \log c-\log (M)) \\
& =2 \frac{\dot{c}}{c}-\frac{\dot{M}}{M} .
\end{aligned}
$$

Finally, substituting $\dot{c}=-v$ yields an extended, parameter free system

$$
\begin{aligned}
\dot{c} & =-v \\
\dot{M} & =-v \\
\dot{v} & =v\left(-2 \frac{v}{c}+\frac{v}{M}\right)
\end{aligned}
$$

As the example illustrates, the states of the parameter free extended system consists of the concentrations $c$, the denominators of the reaction rates $M$ and the reaction rates $v$. The mapping

$$
\Theta: \quad\left[\begin{array}{l}
c \\
p
\end{array}\right] \mapsto\left[\begin{array}{c}
c \\
M(c, p) \\
v(c, p)
\end{array}\right]
$$

is diffeomorph if Assumption 1 holds, and defines a smooth and bijective state-space transformation of the original 


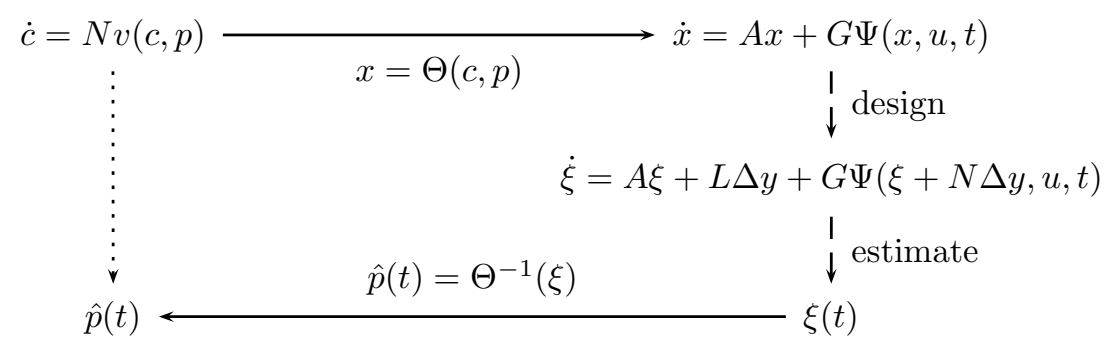

Fig. 1. Overview of the proposed parameter estimation. The dotted line depicts the direct way of estimating the parameter. The proposed approach however, first transforms the system into parameter free coordinates using the mapping $\Theta(\cdot)$. In these coordinates the observer estimates the states (dashed line), where $\Delta y=C \xi-y$ is the error of estimated and measured output. Finally, the parameter estimate is obtained using the inverse transformation $\Theta^{-1}(\cdot)$.

system (3) into the equivalent parameter free extended system:

$$
\begin{aligned}
\dot{c} & =N v+N_{u} u, \\
\dot{M}_{i j} & =\eta_{i j} c_{j}^{\eta_{i j}-1} e_{j}^{T}\left(N v+N_{u} u\right), \\
\dot{v} & =\operatorname{diag}(v)\left(\nu(\operatorname{diag}(c))^{-1}\left(N v+N_{u} u\right)-\tilde{m}\right),
\end{aligned}
$$

where

$$
\tilde{m}_{i}=\sum_{j} \frac{\eta_{i j} c_{j}^{\eta_{i j}-1} e_{j}^{T}\left(N v+N_{u} u\right)}{M_{i j}} .
$$

Here, $u$ denotes the external, measured input. Defining the extended state as

$$
x=\left[\begin{array}{c}
c \\
\operatorname{vect} M \\
v
\end{array}\right]
$$

the system (6) can be compactly written as

$$
\dot{x}=f(x, u)=\left[\begin{array}{c}
f_{c}(c, u) \\
f_{M}(c, v, u) \\
f_{v}(c, M, v, u)
\end{array}\right]
$$

Both the transformation and the transformed system are independent of the parameters. These are now hidden in the initial conditions of the states $M$ and $v$. The parameter free system simplifies the design of an observer. The next section describes the design of a dissipative observer.

\section{DESIGN OF A DISSIPATIVE OBSERVER}

The concept of dissipativity was proposed by Willems [1972a,b] and applied to the observer design by Osorio and Moreno [2006], Moreno [2008]. Its underlying idea is to decompose the nonlinear system into the interconnection of a linear dynamical system $(A, G, F)$,

$$
\begin{aligned}
& \dot{x}=A x+G w, \\
& \tilde{y}=F x=\left[\begin{array}{l}
y \\
\sigma
\end{array}\right]=\left[\begin{array}{l}
C x \\
H x
\end{array}\right],
\end{aligned}
$$

where $y$ denotes the measured and $\sigma$ the unmeasured output, and a static, possibly time-varying nonlinearity

$$
w=\Psi(\sigma, u, t) .
$$

Generally, an observer is a dynamic system that estimates all (unmeasured) states $x$ from the measured output $y$. Moreno [2008] proposed a Luenberger observer for system (9) composed of an copy of the system equations (9) and two additional correction terms feeding back the error of predicted $C \xi$ and measured output $y$

$$
\dot{\xi}=A \xi+G \Psi(\xi+N \cdot(C \xi-y), u, t)+L \cdot(C \xi-y),
$$

where the matrices $L$ and $N$ are design parameters.

For analysing the convergence of the estimate, i.e. $\xi(t) \rightarrow$ $x(t)$ for $t \rightarrow \infty$, it is convinient to look at the error of the estimate $e=\xi-x$. Straight forward calculation shows that the dynamics of the error are given by

$$
\begin{aligned}
\dot{e} & =A_{L} e+G v \\
z & =H_{N} e \\
v & =-\Phi(z, x, u, t),
\end{aligned}
$$

where

$$
\begin{aligned}
A_{L} & =A+L C, \\
H_{N} & =H+N C \\
\Phi(z, x, u, t) & =\Psi(x, u, t)-\Psi(x+z, u, t) .
\end{aligned}
$$

To ensure convergence of the estimate, $e=0$ must be a globally attractive steady state of System (11).

System (11) is a linear dynamical system (11a) with a input dependent, static nonlinear state feedback (11c). From (11f) it is clear that for vanishing error $e_{\mathrm{ss}}=0$ the feedback becomes zero $v=0$, and that $e_{\mathrm{ss}}=0$ is indeed a steady state. This steady state condition holds for any input $u(t)$ and any trajectory $x\left(t, x_{0}\right)$.

To achieve that $e_{\mathrm{ss}}=0$ is a globally stable steady state, we combine a dissipativity condition on the linear part (11a) with a matched dissipativity condition on the nonlinear feedback (11c).

Definition 3. [Osorio and Moreno, 2006]. The nonlinear part of the error dynamics $\Phi$ is called $(Q, S, R)$-dissipative if there exists a non positive semidefinite quadratic form

$$
\omega(\Phi, z)=\Phi^{T} Q \Phi+2 \Phi^{T} S z+z^{T} R z \geq 0,
$$

for all $x, u$ and $t$.

Definition 4. [Osorio and Moreno, 2006]. The error dynamics are called $\left(-R, S^{T},-Q\right)$-state strictly dissipative, if there are matrices $L$ and $N$, a matrix $P=P^{T} \succcurlyeq 0^{1}$ and a scalar $\epsilon>0$ such that

$$
\left[\begin{array}{cc}
P A_{L}+A_{L}^{T} P+\epsilon P+H_{N}^{T} R H_{N} & P G-H_{N}^{T} S^{T} \\
G^{T} P-S H_{N} & Q
\end{array}\right] \preccurlyeq 0 .
$$

1 Throughout this manuscript, the curly symbols $\preccurlyeq$ and $\succcurlyeq$ refer to inequalities in terms of semi-definiteness. 


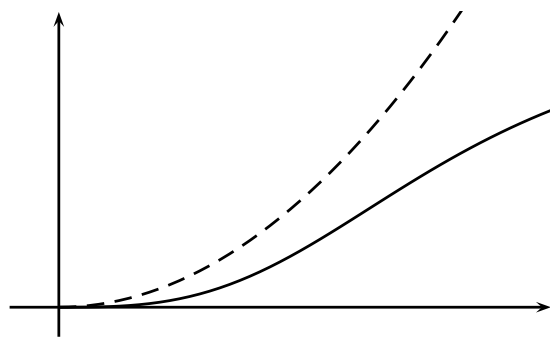

Fig. 2. Bounding $\Phi$ using a quadratic term.

Osorio and Moreno [2006] derived the following theorem, proving exponential convergence of the observer error.

Theorem 5. (Dissipative Observer). Assume that the nonlinearity $\Phi$ is $(Q, S, R)$-dissipative and the linear part $\left(A_{L}, H_{N}\right)$ is $\left(-R, S^{T},-Q\right)$-state strictly dissipative, then the system (4) is a globally exponential observer for the closed loop system (9), i.e. it holds

$$
\|e(t)\|_{2} \leq \sqrt{\frac{\lambda_{\max }(P)}{\lambda_{\min }(P)}}\|e(0)\|_{2} \exp \left(-\frac{1}{2} \epsilon t\right) .
$$

The inequalities (12) and (13) are nonlinear in the unknowns. The next section derives sufficient conditions for solving these inequalities, by transforming them into linear matrix inequalities.

\subsection{Sufficient Conditions for the Dissipative Observer}

To simplify the observer design, the dissipativity conditions are reformulated as linear matrix inequalities (LMIs), which semidefinite programming can solve efficiently.

From (16) it is clear that $R$ needs to be positive semidefinite and $Q$ must be negative semi-definite. To compensate for the mixed term $\Phi^{T} S z, R$ or $Q$ needs to be sufficiently large. A sufficient condition is for example $R>0$ together with

$$
z^{T} R z \geq-\Phi^{T} Q \Phi-2 \Phi^{T} S z .
$$

This condition is satisfied if

$$
\sigma_{\min }^{R}\|z\|_{2}^{2} \geq \sigma_{\max }^{Q}\|\Phi\|_{2}^{2}+2\|S\|_{2} \cdot\|\Phi\|_{2} \cdot\|z\|_{2},
$$

where $\sigma_{\min }^{X}$ and $\sigma_{\max }^{X}$ denote the smallest and largest singular value of $X$ respectively.

From (11f) it is clear that $\Phi(0, x)=0$, and a Taylor expansion of $\Phi(\cdot, x)$ with respect to $z$ gives

$$
\sigma_{\min }^{R}\|z\|_{2}^{2} \geq \sigma_{\max }^{Q} \delta^{2}\|z\|_{2}^{2}+2 \delta\|S\|_{2} \cdot\|z\|_{2}^{2}+\mathcal{O}^{3}(z),
$$

where $\delta$ denotes an upper bound on the slope of $\Phi(\cdot, x)$. Considering the definition of $\Phi$ from (11f) such an upper bound is given by $\delta=\max _{x \in \mathcal{M}}\left\|\frac{\partial}{\partial x} \Psi\right\|$.

Assumption 6. The nonlinearity $\Phi$ is bounded, i.e. it holds that $\max _{z, x}\|\Phi(z, x)\|_{2}<\tilde{\omega}$.

If $\phi$ is bounded, then the higher order terms $\mathcal{O}^{3}(z)$ in (14) are bounded and there exists a constant $\omega>0$ such that with

$$
\sigma_{\min }^{R} \geq \sigma_{\max }^{Q} \delta^{2}+2 \delta\|S\|_{2}+\omega,
$$

the dissipativity condition (12) holds globally (Figure 2).

The matrix inequality A Schur complement on the upper left element of the matrix inequality (13) yields

$$
\left[\begin{array}{ccc}
-R^{-1} & H_{N} & 0 \\
H_{N}^{T} & P A_{L}+A_{L}^{T} P+\epsilon P & P G-H_{N}^{T} S^{T} \\
0 & G^{T} P-S H_{N} & Q
\end{array}\right] \preccurlyeq 0 .
$$

This is a linear matrix inequality in the unknowns $R^{-1}$, $P, Q, L, N$ and $S^{*}=S H_{N}$ Thus, for a given $\epsilon,(16)$ can be solved efficiently using semi-definite programming. In particular, this gives the observer gain matrices $L$ and $N$. Note 2. We can demand $H_{N} \in \mathbb{R}^{n_{x}, n_{x}}$ to be non-singular, and after solving (16), calculate $S$ as $S=S^{*} H_{N}^{-1}=(H+$ $N C)^{-1}$.

Recall that $\omega(\Phi, z)>0$ must also hold, which can be achieved by incorporating (15) as additional constraint for (16). Unfortunately, the two inequalities are coupled in a nonlinear fashion. This is resolved by adding two constraints, first setting $S=0$ and second defining a lower bound for the singular values of $R$. Under these conditions, (15) is equivalent to the following LMIs in the unknowns $Q$ and $R^{-1}$

$$
\begin{aligned}
R^{-1} & \prec \frac{1}{\sigma_{\min }^{R}}, \\
\sigma_{\min }^{R} & \geq\|Q\|_{2} \delta^{2}+\omega .
\end{aligned}
$$

To see this, note that $\left(\sigma_{\min }^{R}\right)^{-1}=\sigma_{\max }^{R^{-1}}$ and that $R$ is symmetric positive definite, i.e. $\sigma_{\max }^{R^{-1}}$ equals the largest eigenvalue of $R^{-1}$.

Summarising, the above considerations derived simple, linear conditions guaranteeing that both nonlinear dissipativity conditions (3) and (3) are satisfied. For convenience these conditions are collected in the following system of linear matrix inequalities:

$$
\begin{aligned}
& P \succ 0, \\
& R^{-1} \prec \frac{1}{\sigma_{\min }}, \\
& \sigma_{\min }^{R} \geq\|Q\|_{2} \delta^{2}+\omega, \\
& {\left[\begin{array}{ccc}
-R^{-1} & H_{N} & 0 \\
H_{N}^{T} & P A_{L}+A_{L}^{T} P+\epsilon P & P G \\
0 & G^{T} P & Q
\end{array}\right] \preccurlyeq 0 . }
\end{aligned}
$$

To solve the system of LMIs, we choose a desired convergence rate $\epsilon$ of the observer and a lower bound $\sigma_{\min }^{R}$ on the singular values of $R$.

Note 3. We set $S=0$ because the nonlinear coupling $S^{*}=H_{N}^{-1}$ prevents us from formulating an upper bound on $\|S\|_{2}$ in terms of a LMI.

Sufficient condition for the existence of a solution for an arbitrarily large $\delta$

Note 4. If the pair (A, C) is observable, then the system of LMIs (18) is solvable for any given $\delta<\infty$. To see this, note that if $(\mathrm{A}, \mathrm{C})$ is observable, then there exists an $L$ such that $A_{L}=A-L C$ is Hurwitz. Then $P A_{L}+A_{L}^{T} P+\epsilon P$ is negative definite. For sufficiently large $P$, the LMI (16) is diagonal dominant. Thus, the LMI (16) is solvable for all $R \succ 0$ and $Q \prec 0$.

\subsection{Dissipative observer for the parameter-free system}

For the extended system (6), a dissipative observer design is very well suited as the system is fully known and can 
be written as a Lur'e system, i.e. the feedback of a linear dynamical and a static nonlinear part. With

$$
H=I, \quad G=\left[\begin{array}{l}
0 \\
I
\end{array}\right],
$$

the system (6) is of the form (9), where in the simplest case, the linear part contains just the stoichiometry

$$
A=\left[\begin{array}{ccc}
0 & 0 & N \\
0 & 0 & 0
\end{array}\right]
$$

and the nonlinear part contains the functions $f_{M}$ and $f_{v}$

$$
\Psi(\sigma, u, t)=\Psi x, u=\left[\begin{array}{l}
f_{M}(x, u) \\
f_{v}(x, u)
\end{array}\right] .
$$

An alternative is to use the Jacobian at some reference point $x_{\text {ref }} A_{\Psi}=\left(\frac{\partial}{\partial x} f\right)\left(x_{\text {ref }}\right)$ wherewith

$$
A=\left[\begin{array}{ccc}
0 & 0 & N \\
& A_{\psi} &
\end{array}\right]
$$

and

$$
\Psi(x, u)=\left[\begin{array}{c}
f_{M}(x, u) \\
f_{v}(x, u)
\end{array}\right]-G A_{\Psi} x .
$$

We can guarantee that Assumption 6 is satisfied as follows. With Assumption 1 follows from the rational form of the reaction kinetics that the norm of $\Psi(x)$ possesses an upper bound for any trajectory $x\left(t, x_{0}\right)$. This is not necessarily true for the observer $\Psi(x+z)$. However, if we bound $\Psi(x+$ $z)$ artificially with $\hat{\Psi}(x+z)$ such that no error is introduced on the true trajectories, i.e. $\hat{\Psi}\left(x\left(t, x_{0}\right)\right)=\Psi\left(x\left(t, x_{0}\right)\right)$, then Assumption 6 holds globally.

\subsection{Calculation of the parameter values}

The final step of the methodology is the actual calculation of the parameter values from the extended state estimate

$$
\left[\begin{array}{c}
\hat{c} \\
\hat{M} \\
\hat{v}
\end{array}\right]=\xi
$$

Using the definition of the reaction kinetics (2b), the parameter estimate is

$$
\begin{aligned}
\hat{K}_{i j}(t) & = \begin{cases}\left(\hat{M}_{i j}(t)-\hat{c}_{j}(t)\right)^{1 / \eta_{i j}} & \text { for } \eta_{i j}>0 \\
1 & \text { for } \eta_{i j}=0 .\end{cases} \\
\hat{k}_{i}(t) & =\hat{v}_{i}(t) \prod_{j}^{n_{c}} \frac{\hat{M}_{i j}(t)}{\hat{c}_{j}(t)^{\nu_{i j}}} .
\end{aligned}
$$

Clearly the parameter estimate is time dependent. It converges to the true, constant values if and only if the observer converges.

\section{EXAMPLE}

The proposed methodology is illustrated using a generic enzyme kinetic model as it appears in many biological systems. A substrate is transformed into a product after forming a complex with an enzyme:

$$
\begin{gathered}
\stackrel{u}{\longrightarrow} S, \\
S+E \stackrel{r_{1}}{\underset{r_{2}}{\rightleftharpoons}} C \stackrel{r_{3}}{\longrightarrow} P, \\
P \stackrel{r_{4}}{\longrightarrow},
\end{gathered}
$$

Parameter and initial conditions that generated the true output $y(t)$

\begin{tabular}{cccc|cccc}
\hline$k_{1}$ & $k_{2}$ & $k_{3}$ & $k_{4}$ & $S_{0}$ & $E_{0}$ & $C_{0}$ & $P_{0}$ \\
1 & 0.1 & 1.5 & 2 & 1 & 9 & 1 & 1
\end{tabular}

\begin{tabular}{cccccccc}
\multicolumn{1}{c}{ Initial conditions of the observer } \\
\hline$\xi_{1}$ & $\xi_{2}$ & $\xi_{3}$ & $\xi_{4}$ & $\xi_{5}$ & $\xi_{6}$ & $\xi_{7}$ & $\xi_{8}$ \\
5 & 5 & 5 & 5 & 5 & 5 & 5 & 5
\end{tabular}

Table 1. Parameter values and initial conditions generating the data shown in Figures 3 and 4 .

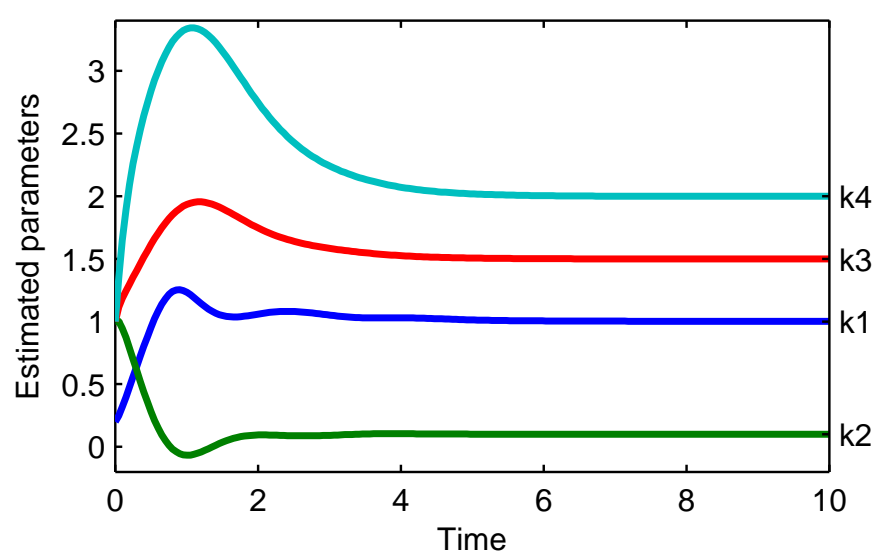

Fig. 3. Estimated parameter values converge nicely to the true values.

where $S$ denotes the substrate, $E$ the enzyme, $C$ the enzyme-substrate complex and $P$ the product. The flow $u$ denotes the input of the system supplying the substrate.

Applying the law of mass action, the corresponding ordinary differential equations are

$$
\begin{aligned}
& \frac{d}{d t}\left[\begin{array}{l}
S \\
E \\
C \\
P
\end{array}\right]=\left[\begin{array}{llll}
-r_{1}+r_{2} & & +u \\
-r_{1}+r_{2} & +r_{3} & \\
+r_{1} & -r_{2} & -r_{3} & \\
& & +r_{3} & -r_{4}
\end{array}\right], \\
& {\left[\begin{array}{l}
r_{1} \\
r_{2} \\
r_{3} \\
r_{4}
\end{array}\right]=\left[\begin{array}{c}
k_{1} S E \\
k_{2} C \\
k_{3} C \\
k_{4} P
\end{array}\right]}
\end{aligned}
$$

where the reaction constants $\left[\begin{array}{llll}k_{1} & k_{2} & k_{3} & k_{4}\end{array}\right]=p$ denote the parameters.

With the following output

$$
y=\left[\begin{array}{lllll}
S & E & C & P & r_{1}
\end{array}\right]^{T},
$$

the pair $(\mathrm{A}, \mathrm{C})$ of the extended system as in (9) is observable. The observability guarantees that the corresponding LMIs can be solved for arbitrarily large $\delta$, ensuring global convergence of the observer.

Simulation studies using artificially generated data shows nice convergence of the state and parameter estimates. Figures 3 and 4 show a representative simulation with true parameter values as in Table 1 . In order to solve the LMIs for the observer the following design specifications were chosen: $\epsilon=0.5, \sigma_{\min }^{R}=1$ and $\delta=500$. As indicated by Figure 4 the presented observer is an exponential observer with a guaranteed convergence rate of $\epsilon$. 

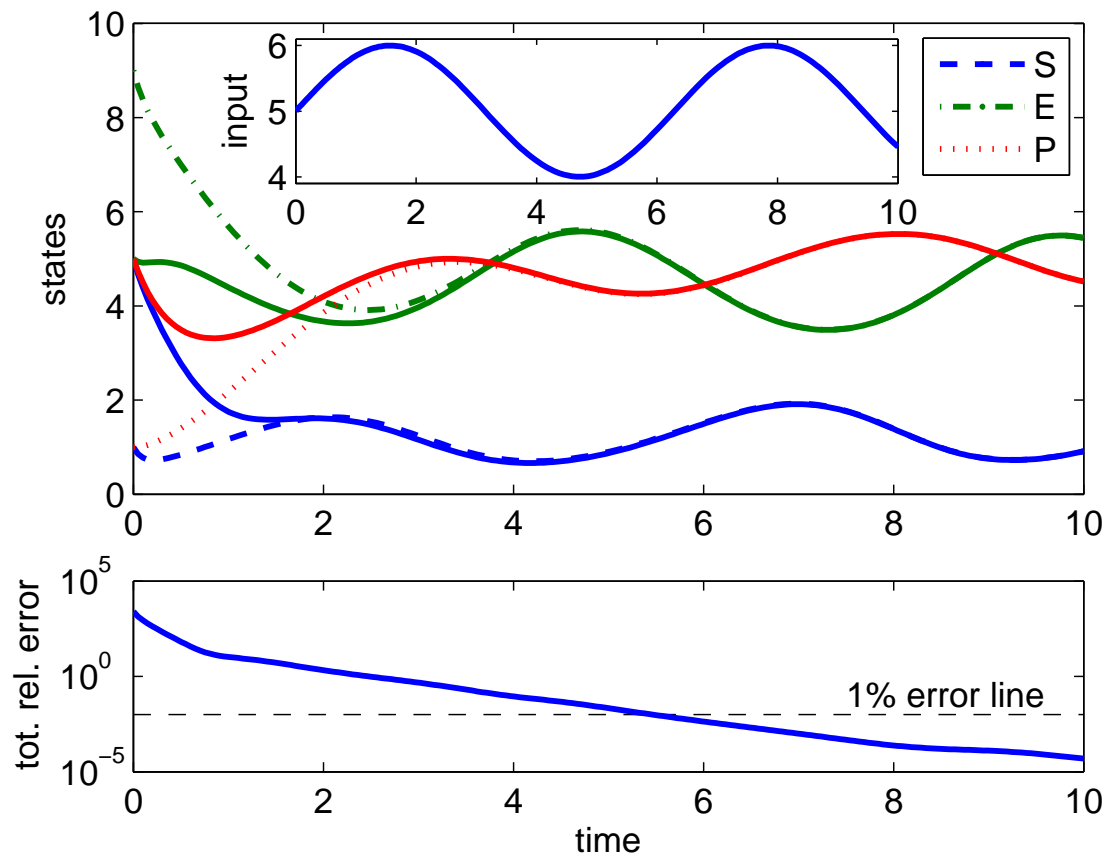

Fig. 4. Top: Comparison of some true (dashed, dash-dotted, dotted) and observed (solid) states. Inlay shows the input. Bottom: Exponential decay of the total relative error $\|(x-\xi) / x\|_{2}$.

\section{CONCLUSIONS}

This paper proposes to recast the estimation of kinetic parameters in biological systems into a state observation problem of an extended system. Using structural information only, an extended system model can be derived that is independent of the parameters and in Lur'e form. This special structure is advantageous for using a dissipative observer approach. Several sufficient conditions are presented that simplify the design of a dissipative observer. The applicability of the methodology is illustrated at the example of a enzyme kinetic model.

\section{REFERENCES}

A. Cornish-Bowden. Fundamentals of Enzyme Kinetics. Portland Press, third edition, 2004.

M. Farina, R. Findeisen, E. Bullinger, S. Bittanti, F. Allgöwer, and P. Wellstead. Results towards identifiability properties of biochemical reaction networks. In Proc. of the 45th IEEE Conf. on Decision and Control, San Diego, USA, pages 2104-2109, 2006.

D. Fey, R. Findeisen, and E. Bullinger. Parameter estimation in kinetic reaction models using nonlinear observers is facilitated by model extensions. In 17th International Federation of Automatic Control World Congress, Seoul, Korea, pages 313-318, 2008.

D. Fey, R. Findeisen, and E. Bullinger. Identification of biochemical reaction networks using a parameter-free coordinate system. In Pablo A. Iglesias and Brian Ingalls, editors, Control-Theoretic Approaches in Systems Biology. MIT press, 2009. in print.

A. L. Hodgkin and A. F. Huxley. A quantitative description of membrane current and its application to conduction and excitation in nerve. J Physiol, 117(4): 500-544, 1952.
J. Keener and J. Sneyd. Mathematical Physiology, volume 8 of Interdisciplinary Applied Mathematics. Springer-Verlag, New York, second edition, 2001.

E. Klipp, R. Herwig, A. Kowald, C. Wierling, and H. Lehrach. Systems Biology in Practice: Concepts, Implementation and Application. Wiley-VCH, Weinheim, 2005.

J. A. Moreno. Observer design for bioprocesses using a dissipative approach. In 17th International Federation of Automatic Control World Congress, Seoul, Korea, 15559-15564, 2008.

M. Osorio and J. A. Moreno. Dissipative design of observers for multivalued nonlinear systems. In Proc. of the 45th IEEE Conf. on Decision and Control, San Diego, CA, USA, pages 5400-5405, 2006.

Eduardo Sontag. Molecular systems biology and control. European J Control, 11(4-5):396-435, 2005.

J. C. Willems. Dissipative dynamical systems - Part I: General theory. Archive for Rational Mechanics and Analysis, 45(5):321-351, 1972a.

J. C. Willems. Dissipative dynamical systems - Part II: Linear systems with quadratic supply rates. Archive for Rational Mechanics and Analysis, 45(5):352-393, 1972b. 